\section{Myeloid/T-cell acute lymphoblastic leukemia in children and adults}

\author{
Sabina Chiaretti, Monica Messina, \\ Simona Tavolaro, Robin Foà \\ Division of Hematology, Department \\ of Cellular Biotechnologies and Hematology, \\ Sapienza University, Rome, Italy
}

\begin{abstract}
Until recently, few molecular aberrations were recognized in T-cell acute lymphoblastic leukemia (T-ALL) and they were restricted to aberrations involving the T-cell receptor (TCR). The introduction of powerful technologies has allowed to identify novel rearrangements. In this context, we have performed a gene expression profiling analysis on a relatively large cohort $(n=69)$ of adult patients with a diagnosis of T-ALL. By unsupervised clustering, we identified 5 subgroups. Of these, one branch included 7 patients (10\%) whose gene expression profile resembled that of AML. These cases were characterized by the overexpression of a large set of myeloid-related genes, as well as of miR-223. Finally, these patients appear to have an unfavorable clinical course. This newly identified subset of T-ALL cases partly resembles the so-called ETP (early T-precursor) pediatric subgroup: both age groups have in fact a peculiar gene expression profile, an unfavorable outcome and an incidence of about $10 \%$.
\end{abstract}

T-cell acute lymphoblastic leukemia (T-ALL) is a malignancy of T-cell precursors, present in about $15 \%$ of children and $25 \%$ of adults. Up to the past decade, few events were recognized in T-ALL, with biologic knowledge being generally restricted to aberrations involving the T-cell receptor (TCR). The scenario has been recently rapidly evolving: the most common aberrations currently include i) overexpression of known ongogenes, such as TLX1 and TLX3; ii) mutations of NOTCH1, FBXW7, JAK1, WT1 and PTEN; iii) rearrangements, that include SIL/TAL1, NUP214/ABL1, EML/ABL1, CALM/AF10 and TAF_I-NUP214; iv) finally, amplifications and deletions have been documented, the most frequent involving MYB, CDKN2A and RB1.

The identification of these aberrations has led to pinpoint cellular functions that are specifically deregulated, such as cell cycle control, cell differentiation, proliferation and/or survival and self-renewal properties. In this context, gene expression analysis has proven useful in recognizing signatures that are associated with the overexpression of known oncogenes, with the differentiative stage of the leukemic cell, with the recognition of novel subgroups, as well as with outcome.

A broad use of gene expression profiling (GEP) has also permitted to identify distinct subsets of patients and to recognize similarities between different subsets and/or diseases. In acute myeloid leukemia (AML), a subset of cases whose genomic profile is similar to that of CEBPA mutated patients was previously described: these patients have a peculiar profile that is characterized by the overexpression of a set of T-lineage genes, NOTCH1 mutations and hypermethylation of the CEBPA promoter, suggesting that the combination of these lesions may lead to a mixed T-lineage/myeloid lineage scenario.

By analyzing the gene expression profile of 69 samples of newly diagnosed adult T-ALL, we have identified, by unsupervised analysis, 5 distinct subgroups: for the majority of them the underlying lesion had been previously identified. In addition, we identified a novel subgroup, defined myeloid-like, that revealed a very peculiar profile and was characterized by the overexpression of several myeloid-related genes, such as genes codifying for myeloid antigens, myeloid transcription factors (including CEBPA) and granule proteins. These cases were also characterized by high expression levels of IL-8. Notably, an unsupervised clustering carried out in a series of cases that included also 19 AML samples branched these cases with the AML patients, corroborating the strict similarity between the two subsets of patients. Interestingly, these cases did not have peculiar features at the immunophenotypic level. Quantitative PCR experiments confirmed the high expression levels of a set of these genes, such as CEBPA, CEBPB, MPO and IL-8, the latter being previously reported to be associated with refractoriness to induction chemotherapy. Thus, in order to understand the underlying mechanism responsible for this characteristic signature and taking advantage of the evidence that in AML hypermethylation of the CEBPA promoter region appears responsible of the mixed T-lineage/myeloid lineage, we wondered if CEBPA methylation could play a role also in our T-ALL series: we evaluated the CEBPA promoter-CpG island methylation status in 7 classic T-ALL cases and in 4 myeloidlike patients. Our analysis revealed that only 1/7 T-ALL samples was methylated, while 2/4 myeloid-like patients were methylated despite the high levels of CEBPA expression, thus indicating that CEBPA expression levels are not correlated with the methylation status of its promoter and that other mechanisms might regulate CEBPA expression in T-ALL.
Correspondence: Sabina Chiaretti, Division of Hematology, Department of Cellular Biotechnologies and Hematology, Sapienza University of Rome, Via Benevento 6, 00161 Rome, Italy. Tel: +39-06-441639824 - Fax: +39-06-85795792. E-mail: chiaretti@bce.uniromal.it

Key words: T-cell acute lymphoblastic leukemia.

Received for publication: 4 May 2011.

Accepted for publication: 4 June 2011.

This work is licensed under a Creative Commons Attribution NonCommercial 3.0 License (CC BYNC 3.0).

(C) Copyright S. Chiaretti et al., 2011

Licensee PAGEPress, Italy

Pediatric Reports 2011; 3(s2):e3

doi:10.4081/pr.2011.s2.e3

Considering that miR-223 regulates myeloid differentiation, we subsequently evaluated the expression of this miR: this analysis was carried out on 5 myeloid-like patients, 12 T-ALL patients, 8 AML patients and $3 \mathrm{CD} 2+$ samples obtained from healthy donors. The results obtained showed that "myeloid-like patients" have expression levels of miR-223 comparable to those of AML and significantly higher than those of the other T-ALL.

Finally, we evaluated the clinico-biologic features of these patients: there was not a specific differentiative block in these cases, as well as no peculiar immunophenotypic characteristic; similarly, the clinical presentation was not different from that of the remaining T-ALL cases. At variance, the outcome of these patients was poor: in fact, of the 7 patients 2 succumbed during induction chemotherapy, 2 were refractory to induction chemotherapy and only 2 obtained a complete remission, with 1 patient relapsing shortly after.

The "myeloid-like" group in some way resembles the ETP (early T-precursor) subgroup recently identified in pediatric T-ALL. In fact: i) the incidence of myeloid-like and ETP is of $10 \%$ and $12 \%$ of cases in adult and pediatric cohorts, respectively; ii) both groups retain a certain degree of multilineage differentiation potential; iii) both cohorts have been associated to an unfavorable clinical outcome.

In conclusion, this work allowed to define a novel subgroup of adult T-ALL that was not recognized by other standard approaches, underlining the importance of gene profiling in further dissecting acute leukemia subsets. The recognition of this subgroup may also have clinical, prognostic and therapeutic implications, since the outcome of this set of patients appears to be particularly poor, and suggests 